\title{
Model Uncertainty and Energy Technology Policy: The Example of Induced Technical Change \\ Yongyang $\mathrm{Cai}^{\dagger} \quad$ Alan H. Sanstad ${ }^{\S}$
}

July 2015

\begin{abstract}
Numerical modeling based on economic principles has become the dominant analytical tool in U. S. energy policy. Energy models are now used extensively by public agencies, private entities, and academic researchers, and in recent years have also formed the core of "integrated assessment" models used to analyze the relationships among the energy system, the economy, and the global climate. However, fundamental uncertainties are intrinsic in what has become the typical circumstance of multiple models embodying different representations of the energy-economy, and producing different policy-relevant outputs that model users are compelled to interpret as equally plausible and/or valid. Because the policy implications of these outputs can diverge substantially, policy-makers are confronted with a significant degree of model-based uncertainty and little or no guidance as to how it should be addressed.

This problem of "model uncertainty" has recently been the focus of work in macroeconomics, where scholars have studied the problem of how a decision-maker should proceed in the face of uncertainty regarding the correct model of an economic system that is the object of policy. A unifying theme in this work is the identification of decision-rules that are robust to such uncertainty. This paper describes an application to energy modeling of the macroeconomists' insights and methods related to model uncertainty and robust analysis, focusing on the important example of model representations of technical change. Using a well-known model by Goulder and Mathai, we treat contrasting assumptions on technical change - and their implications for $\mathrm{CO} 2$ emissions abatement policy - as a phenomenon of model uncertainty. We apply a non-Bayesian decision rule - so-called "min-max regret" - to this problem and computationally solve the model under the min-max regret criterion, yielding a policy - an emissions abatement path - that reflects a form of robustness to the model uncertainty.
\end{abstract}

JEL Classification: Q54, D81, C63

Keywords: Energy and climate policy; technical change; model uncertainty; min-max regret; robust analysis

* This paper is adapted from a working paper version released by the Center for Robust Decision-Making on Climate and Energy Policy (RDCEP) at the University of Chicago. This research was supported by the National Science Foundation (SES-0951576) through RDCEP, and Mr. Sanstad's work was supported by the U. S. Department of Energy under Lawrence Berkeley National Laboratory Contract No. DE-AC02-05CH11231. We would like to thank William Brock, Kenneth Judd, Todd Munson, and our other RDCEP colleagues for their comments, and two anonymous referees for very useful comments and suggestions.

${ }^{\dagger}$ Hoover Institution at Stanford University \& Becker Friedman Institute at University of Chicago, yycai@stanford.edu.

${ }^{\S}$ Corresponding author: Berkeley, CA. ahsanstad@lbl.gov. 


\section{Introduction}

Over the past four decades, numerical modeling based upon economic principles has become the dominant analytical tool in U. S. energy policy. Models of the energy system or sub-systems, of the national economy with emphasis on energy sectors, and combinations of these two types have both proliferated in number and increased in complexity and detail. They are now used by regulatory agencies, university researchers, private companies and non-profit organizations. Moreover, in recent years numerical energy-economic models have formed the core of "integrated assessment" models that represent the relationships among the economy, the energy system, and the global climate. ${ }^{1}$

Their usefulness notwithstanding, however, the widespread application of numerical energy-economic models in policy analysis poses certain challenges for decision-makers. Among these is the situation of multiple, "co-existing" models embodying what amount to competing representations of the energy-economy, and producing different policy-relevant outputs. While structured multi-model scenario analyses are a well-established methodology in the energy modeling community, this community does not provide formal or quantitative model rankings. As a consequence, results from a now sizable group of models must often be interpreted by users as equally plausible and/or valid. Given that the policy implications of these results can diverge substantially even in structured comparisons, this circumstance confronts policy-makers with a significant degree of uncertainty and little or no guidance as to how it should be addressed.

The aim of this paper is to demonstrate how this type of fundamental model uncertainty can be represented and analyzed in the context of one of this era's most important energy policy problems: determining optimal strategies for reducing carbon dioxide (CO2) emissions from the energy sector that contribute to global climate change. We focus on a particularly significant dimension of model uncertainty: the representation of technological change, in this case of the type that lowers the cost of $\mathrm{CO} 2$ emissions abatement. Our approach is to apply a non-Bayesian decision rule and solution concept to a model that incorporates the key mechanism while being sufficiently simple to clearly exemplify the analytical approach and provide insight into the results.

The importance of fundamental energy and integrated assessment model uncertainty, and the practical implications of not addressing it, were noted by Fischer and Morgenstern [1] in their study of the divergence of model-based estimates of the potential costs to the U. S. economy of the Kyoto carbon emissions reduction agreement. These estimates varied by a factor of five. As these authors pointed out, “...this variability in cost estimates undermines support for mandatory policies to curb emissions, as policy makers are generally reluctant to adopt a major program without an understanding of its true costs."

Not all multi-model, policy-relevant outputs in energy analysis display this level of variation. Nonetheless, inter-model differences large enough to be policy relevant are the norm

\footnotetext{
${ }^{1}$ We will use the term "energy model" to refer to each of these types, i.e., numerical economic equilibrium (partial or general) or optimization models, with or without linked environmental components.
} 
rather than the exception. Decision-makers may reasonably infer that such "ensemble uncertainty" accurately reflects the present-day limits of our ability to predict the consequences of large-scale energy or environmental policy. If so, then the problem of rationally using multimodel policy outputs should be addressed in its own right.

In macroeconomics, this problem of model uncertainty has been the focus of work by Hansen and Sargent [2],[3],[4] and Brock et al. [5], [6], [7]. These scholars have studied the problem of how a decision-maker should proceed in the face of uncertainty regarding the correct model of an economic system that is the object of policy. A unifying theme in this work is identification of decision-rules that are "robust" to such uncertainty. While there are different technical definitions of this concept, colloquially it refers to decisions, or policies, that will yield acceptable although not necessarily optimal outcomes regardless of which model within a certain set is "true."

This paper is based on the observation that, for the reasons described above, this form of uncertainty characterizes the present state of energy modeling, and that the macroeconomists' insights and methods are applicable and can yield important insights. Our focus on technical change is motivated by the long-standing recognition by both experts and non-specialists that assumptions regarding the determinants and dynamics of technical change are a primary driver of model-based projections of the feasibility, costs, and outcomes of long-run energy policies especially those aimed at reducing $\mathrm{CO} 2$ emissions from the energy sector. Among current energy models, quite different sets of such assumptions are maintained - i.e., in different models - and they have divergent policy implications. Broadly speaking, there are two paradigms for representing technical change. In the "autonomous" representation, which can be traced back to Solow's work on aggregate productivity in the 1950s, technical change dynamics are determined exogenously to the market economy [8]. Moreover, while these dynamics may be influenced by government policy, the mechanisms of this influence are left unspecified. By contrast, "endogenous" or "induced" technical change refers to theories, and their numerical implementations, in which technical change is explicitly treated, albeit in simplified form, as an outcome of choices by economic agents acting within markets; in certain examples, this paradigm also allows for the representation of government influences such as R\&D funding.

As might be expected, these two approaches have quite different theoretical and quantitative implications for energy policy. Yet - even after decades of basic and applied research - there is an absence of consensus within the energy modeling community regarding the appropriate paradigm for representing technical change, reflected in a continuing divergence among different numerical models. The departure point for this paper is the observation that this state-of-affairs is best characterized as one of fundamental model uncertainty, and as such can in principle be addressed by bringing to bear the appropriate concepts and tools developed in macroeconomics.

As noted above, the modeling community does not quantitatively rank or assign weightings to sets of models. This state-of-affairs can be thus be viewed as one of "Knightian uncertainty," which refers to uncertainty that cannot be readily described by probabilities. This perspective also underlies the pioneering contribution of Lempert et al. (e.g., McInerney, Lempert, and 
Keller [9]) to the analysis of robust decision-making in integrated assessment modeling, which is one of our inspirations.

In this paper, we follow the modeling paradigm reflected in Brock et al. [5], [6], [7] and in Hansen and Sargent [2], [3], [4], which is to employ models that are sufficiently simple that they can be thoroughly analyzed and can facilitate understanding of the basic concepts. This perspective also reflects the view that, in the words of a prominent energy modeler, "the purpose of energy modeling is insight, not numbers" (Hogan [10]), which became a widely-accepted precept in the modeling community (e.g., Peace and Weyant [11]).

The paper is organized as follows. In the next section, we sketch the history and key concepts of model uncertainty and validity in the energy analysis and policy field. We then further discuss the representation of technological change in energy models and its policy implications. Against this background, we present a model of Goulder and Mathai [12] that, while relatively simple, nevertheless allows for analysis of several fundamental issues associated with differing technical change assumptions and how they affect model-derived policy conclusions. We briefly discuss technical aspects of the model and the key conclusions reached by Goulder and Mathai. Next, we consider the Goulder-Mathai framework from the perspective of model uncertainty, and, following Brock et al. [6], introduce two decision rules - min-max and min-max regret - that are applicable in the context of this form of uncertainty. We briefly review previous and recent applications of min-max regret in energy and integrated assessment modeling. We then describe a computational version of the model and discuss its solution under the min-max regret criterion, comparing this to solutions based on expected cost minimization. The paper ends with a summary and concluding remarks.

\section{$2 \quad$ Validity and uncertainty in energy modeling}

As noted in the introduction, the concept of model uncertainty entails multiple models of a given system being assigned equal weight, credibility, or validity, whether explicitly or - as in the case of energy modeling - implicitly. The prevalence of this form of uncertainty raises the question of why some form of validation procedure cannot be applied to compare and ideally rank models in terms of their likelihood. The answer to this question involves the history and development path of this area of modeling.

The 1970s saw the emergence of numerical energy modeling based on optimization or equilibrium principles with an explicitly microeconomic perspective (Murphy and Shaw [13]). Among their other features, such models allowed for the prospective analysis of hypothetical policies much more readily than was the case in a more standard forecasting approach. At the same time, however, in contrast to what was then the standard approach to, for example, macroeconomic modeling, this form of deterministic "system simulation" modeling generally did not employ econometric estimation techniques for assigning values to model parameters (Greenberger et al. [14]). Thus, concepts of validity based upon the empirical grounding of models in data through classical statistical procedures were for the most part not directly applicable. 
Nevertheless, during this period numerical model validation was an active area of research and application; indeed, this may have reflected the greater complexity of the problems of validation in the simulation paradigm. A 1978 bibliography on validation of computer models in policy analysis and the social sciences contained over seven hundred entries, with more than one-hundred in the category of "energy and electric power models" (Gruhl and Gruhl [15]). More generally, model "assessment" - both "evaluative" and "non-evaluative" (e.g., model intercomparisons) - received substantial attention as well as resources (Greenberger and Richels [16]). For example, the U. S. National Bureau of Standards convened multi-disciplinary workshops on energy model validation [17].

Subsequently, however, these efforts attenuated. In the succeeding decades, a certain tension developed in energy modeling with respect to criteria for validity and likelihood. Experiences in the policy arena in the 1970s, including often difficult problems of communication with decision-makers, led to the emergence among energy modelers of the philosophy, noted above, of the rationale for energy modeling being to generate insight into important issues, rather than numerical results per se [10], [11]. For example, this perspective became the guiding principle for Stanford University's Energy Modeling Forum (EMF), which pioneered, and continues to implement, the methodology of structured energy model intercomparison analyses (Huntington et al. [18]). At the same time, however, the path of actual model development and evolution has shown a pronounced emphasis on greater detail and complexity, to an extent indicating that increased model detail per se is seen as improving likelihood. In many cases, this detail is in the representation of energy technologies, and the resulting change in model output hinges on the particular assumptions made regarding specific technology characteristics - that is, the numbers describing technologies and their role in the energy system and its response to policy.

Both of these philosophies are closely related to the use of calibration rather than estimation procedures for parameterizing energy models. "Calibration" here refers to the use of external sources to assign values for elasticities, rates of technical change, technology cost, and other parameters. As described by Dawkins et al. [19], under the calibrationist philosophy, the validity of numerical models is based on their theoretical underpinnings rather than their empirical likelihood. ${ }^{2}$ This philosophy helps to explain the prevalence of energy model uncertainty. Economic theory supports a wide range of practical choices regarding numerical model structure, functional forms, and other features, and reliance on calibration in turn allows for a range of equally plausible numerical realizations conditional on such features having been selected. Thus, different models can yield "insights" that, all else being equal, are equally credible.

\footnotetext{
2 “.....modelers typically see their simulations largely as numerical implementations of theoretical structures. To them, the widespread use of a particular structure in the theoretical literature is an indication of its worth, so that they seek less to test or validate models and more to explore the numerical implications of a particular model, conditional on having chosen it...." ([19], p. 3762).
} 
While the majority of energy models are deterministic, there have been models that take an explicitly decision-making-under-uncertainty approach (Kann and Weyant [20]). In general, these also reflect the philosophy we have just described, in that the uncertainty is on the part of agents within the model, and is conditional upon prior assumptions regarding model structure and basic parameterization choices. Addressing model uncertainty in the sense defined above, Kann and Weyant [20] proposed a framework for applying uncertainty analysis to deterministic models to elucidate, e.g., the sources of inter-model differences. This framework has not been adopted among energy modelers, however, and well-known reasons for inter-model output differences, such as contrasting choices pertaining to structure and parameter values, continue to be acknowledged qualitatively without being quantified or analyzed in depth. (See, for example, Clarke et al. [21]) The study of Fischer and Morgenstern [1] is a rare exception that proves this rule; they performed a regression analysis in an attempt to quantitative gauge the importance of various model features in determining inter-model output variation. More recently, the U. S. Congressional Research Service conducted an insightful and illuminating investigation of multiple models' projections of the costs of long-run $\mathrm{CO} 2$ abatement policy (Parker and Yacobucci [22]); this analysis is in effect an effort by the U. S. Congress to address energy model uncertainty in the context of a major policy issue.

\section{Modeling energy-related technological change}

Early energy modeling studies emphasized the importance of the mechanisms of substitution i.e., between energy and non-energy economic factors, including capital - in determining the role of energy in the aggregate economy (Hogan and Manne [23]). However, events of the 1970s appeared to demonstrate the importance of overall energy productivity in a manner analogous to that of labor productivity. That is, consider a stylized economy-wide production function $F(\square)$ giving gross output $Y_{t}$ as a function of capital $K_{t}$, labor $L_{t}$, energy $E_{t}$, and factor-augmenting technical change:

$$
Y_{t}=F\left(\alpha_{t}^{K} K_{t}, \alpha_{t}^{L} L_{t}, \alpha_{t}^{E} E_{t}\right)
$$

If $d \alpha_{t}^{i} / d t>0$, then over time, all else being equal, a given input level of factor $i$ will yield a progressively greater level of output. Having been proposed for macroeconomic labor-output relationships by Solow and Swan in the 1950s, this type of relationship suggested itself to explain the aggregate "de-coupling" between energy and economic growth that occurred in the '70s (Solow [8], Swan [24]).

This hypothesis was not completely uncontroversial; Hogan and Jorgenson [25] argued that, on the contrary, the de-coupling phenomenon could be explained in terms of aggregate substitution between energy and other factors, resulting from energy price shocks. Nonetheless, the need to calibrate numerical energy-economic models to observed trends, combined with the simplicity of factor-augmentation, gave rise to widespread adoption of this type of 
representation. Colloquially, the term "autonomous energy efficiency index" or "AEEI" came into common use to refer generically to parameters such as $\alpha_{t}^{E}$ above. Variations on this mathematical representation were also developed for models without production function-based structure.

That this approach was in effect a reduced form for the complex, not-well-understood dynamics of energy-related technical change was generally if tacitly recognized. However, the advent of the "new growth theory" as developed by Romer [26], 27], Lucas [28] and others in the 1980s and 1990s indirectly weakened its plausibility. The new growth theory insight that technological innovation is to a significant extent a market phenomenon, responsive to economic incentives, and its practitioners' development of mathematical tools to represent it in aggregate models, provided grounds for questioning the standard autonomous technical change assumptions in energy models as well as indicating how these assumptions might be modified.

While this issue entails a number of challenging theoretical and empirical problems, its policy relevance is quite intuitive. The fundamental policy application of energy models is to project how government actions such as GHG emissions abatement measures will affect the energy system and the economy. Such actions are generically of several types. The first is direct regulation such as technology adoption requirements. In a literal sense, this is not an "autonomous" change, although depending upon the level of model detail, among other considerations, it can be approximately captured by adjusting AEEI-type parameters. In equilibrium models, however, this technique raises questions of interpretation, since it could imply that the government can simply impose productivity increases in the economy. The second type is promotion of energy-related technical change through government or governmentsponsored R\&D. Here again, the assumption of autonomous technical change poses a prima facie hurdle, since R\&D is fundamentally a production relationship between inputs of factors such as scientific or engineering expertise, and outputs such as patents or actual technologies.

The third type of government action is market- or price-based interventions - especially, emissions taxes or emissions cap and trade regimes. In a model with autonomous technical change, the only possible response to a perturbation such as an emissions tax is substitution away from emissions-intensive energy sources on the part of consumers and firms - technical change is by assumption unaffected. In particular, the possibility that energy price changes might result in energy-or emissions-reducing innovation is ruled out. Such innovation could arise, for example, from private firms undertaking $R \& D$ aimed at creating technologies that would yield profits given a price on emissions. Given that such "induced" or "endogenous" innovation or technical change would yield technologies enabling emissions abatement (for example) at lower cost than existing technologies, the omission of such price-driven innovation would arguably result in systematic over-estimation of the costs of policies.

Over the past fifteen years, research on induced technical change (ITC) in energy models has proliferated; Gillingham et al. [29] is a thorough and insightful review. A number of new computational models have been created incorporating representations of ITC, such as Buonanno et al. [30] and Popp [31]. Underlying principles have been analyzed, including the cost-bias problem with AEEI-based models (Pizer and Popp [32], Popp [33]). This activity has not, 
however, resulted in either a convergence of energy modeling approaches to ITC nor, perhaps more importantly, in changes in the treatment of technical change in established models. That is, the use of AEEI-type representations remains common, and in particular continues in the most firmly established (and influential) energy as well as integrated assessment models in the U. S.

\section{A simple integrated assessment model}

In a widely-cited paper, Goulder and Mathai [12] presented an elegant theoretical framework to capture essential features of the relationship between autonomous and induced technical change, and the consequences of technical change assumptions for optimal policies of $\mathrm{CO} 2$ abatement, in a partial equilibrium setting. They analyzed cases based on both a cost-effectiveness criterion applied to meeting a $\mathrm{CO} 2$ concentration target, and a cost-benefit criterion when abatement costs are weighed against damages from climate change. In each of these two cases, they further distinguished between technical change induced by R\&D investment as well as by learning-bydoing. Among models that have appeared in the literature dealing with technical change and $\mathrm{CO} 2$ emissions abatement, the G-M is distinguished by the combination of being both sufficiently simple to transparently represent key mechanisms of the economics of CO2 abatement and technical change, and powerful enough to allow their exploration in a manner that yields insights. These two aspects are reflected by the influence that the model and G-M's analysis have had in the literature. For these reasons, it is an ideal framework for our purposes.

To adapt the Goulder-Mathai (hereafter, G-M) framework to study model uncertainty, we will focus specifically on the case of cost-benefit analysis with learning-by-doing. The model is as follows. The setting is deterministic, continuous-time, infinite-horizon optimal control. In the absence of abatement, $\mathrm{CO} 2$ emissions are assumed to follow an exogenously given baseline time path $E_{t}^{0}$. Emissions contribute to an atmospheric carbon stock $S_{t}$, resulting in damages $D\left(S_{t}\right)$. Emissions abatement $A_{t}$ can be undertaken at a cost $C\left(A_{t}, H_{t}\right)$ that depends jointly on $A_{t}$ and a stock of "abatement knowledge" $H_{t}$. This cost function is assumed to be twice continuously differentiable, strictly increasing in abatement, and strictly decreasing in knowledge, and is also

assumed to be strictly convex in $A_{t}, \partial^{2} C / \partial A_{t}^{2}>0$, and to exhibit decreasing marginal costs of abatement with respect to knowledge, $\partial^{2} \mathrm{C} / \partial A_{t} \partial H_{t}<0$.

The atmospheric carbon stock follows a standard linear decay model, augmented by baseline emissions and abatement:

$$
\frac{d}{d t} S_{t}=-\varepsilon S_{t}+E_{t}^{0}-A_{t}
$$

with initial condition $S_{0}=S(0)$. The damage function $D\left(S_{t}\right)$ is assumed to be strictly increasing and strictly convex. 
The dynamics of technical change are characterized as follows. A knowledge function $\Psi\left(H_{t}, A_{t}\right)$, increasing in both $H_{t}$ and $A_{t}$, captures the idea that undertaking abatement results in increased knowledge about abatement. This function is weighted by a parameter $\kappa$ and combined with a term representing standard autonomous, in this case knowledge-increasing, technical change, giving the equation-of-motion for the knowledge stock

$$
\frac{d}{d t} H_{t}=\alpha H_{t}+\kappa \Psi\left(H_{t}, A_{t}\right),
$$

with $\alpha>0$ and initial condition $H_{0}=H(0)$. The parameter $\kappa$ controls the influence of induced technical change: $\kappa=0$ represents the case of autonomous change, and $\kappa>0$ induced. G-M treat $\kappa$ as a continuous parameter in their theoretical analysis, while in their numerical examples focus on the two cases $\kappa=0, \kappa=1$.

The decision criterion is to minimize the present value (discounted) cost of abatement plus damages. Thus, the complete G-M model in this case is

$$
\begin{aligned}
& \min _{A_{t}} \int_{0}^{\infty}\left[C\left(A_{t}, H_{t}\right)+D\left(S_{t}\right)\right] e^{-\rho t} d t \\
& \text { s.t. } \\
& \frac{d}{d t} H_{t}=\alpha H_{t}+\kappa \Psi\left(H_{t}, A_{t}\right) \\
& \frac{d}{d t} S_{t}=-\varepsilon S_{t}+E_{t}^{0}-A_{t} \\
& H_{0}, S_{0} \text { given } \\
& A_{t}, H_{t}, S_{t} \geq 0 .
\end{aligned}
$$

G-M derive first-order optimality conditions for this model to analyze the implications for the cost-minimization problem of assuming ITC compared with solely autonomous technical change. By simple inspection, it is clear that the presence of ITC - represented by $\kappa>0$ decreases the cost of abatement. This is because the cost function is decreasing in knowledge capital $H_{t}$ - i.e., $\partial C / \partial H_{t}<0$ - and it is assumed that there are no costs associated with the learning effect. ${ }^{3}$ Moreover, assuming that damages are a convex function of the carbon stock, the time path of the optimal tax falls with the introduction of ITC. In addition, ITC has an ambiguous effect on initial abatement $A_{0}$, but increases the level of cumulative abatement over the entire time horizon.

\section{Extension to model uncertainty analysis}

The G-M analysis can be viewed in a stylized sense as exemplifying the modeling methodology described above, in which multiple models exist to describe the same system or phenomenon. To

\footnotetext{
${ }^{3}$ With additional assumptions, the sign and magnitude of the cost reduction resulting from ITC can also be inferred by applying a dynamic envelope theorem (LaFrance and Barney [35]).
} 
do so, we interpret the parameter $\kappa$ as an index determining a family of models. The two values $\kappa=0$ and $\kappa=1$ succinctly define "no ITC" and "ITC" cases, but any $\kappa>0$ defines a model with some level of ITC. Absent any other information, this perspective abstractly characterizes the circumstance of multiple models yielding different policy conclusions, but in effect assigned equal plausibility by the modeler. Thus, the results in each case would be informative to a hypothetical decision-maker, but the manner in which they might actually be used for decisionmaking is left unspecified. That is, imagine in the present case that an individual or entity sought to decide upon an intertemporal policy of abatement and investment using the G-M model, including the dimension of parametric ITC. How should this be done?

One approach would be to assign a probability distribution to $\kappa$, and convert the problem into one of expected cost minimization. The problem is how to determine an appropriate distribution, or put differently, how to generate priors for the value of this parameter. As discussed above, years of research on ITC in general in energy-environmental modeling have not resulted in consensus regarding the important issues - how it should be modeled, the values of key parameters, and so forth. With a particular model such as G-M's, it would seem possible to conduct empirical (econometric) analysis to quantify $\kappa$. In practice, however, this has proven exceptionally difficult. ${ }^{4}$ Even the standard approach of calibration (as opposed to estimation) of numerical models is challenging in the case of ITC, and moreover a calibration approach would not provide stochastic information to support assignment of a probability distribution.

The conceptual and practical difficulties associated with determining model priors are among the issues discussed by Brock et al. [6] in motivating a non-Bayesian approach to dealing with model uncertainty (in their analysis, in a macroeconomic context). Non-Bayesian decision rules allow a policy-maker to explicitly incorporate multiple models or model specifications without needing to specify probability distributions, or specific probability magnitudes. While individual models may be of optimization type, the overall decision problem is not - instead the goal is to make decisions that will yield at least acceptable outcomes irrespective of which candidate model may be correct - this is a form of robustness to model uncertainty.

Both the technical model details and the economic policy issues studied by Brock et al. differ from those associated with the G-M analysis. Nevertheless, the underlying idea of fundamental model uncertainty and non-Bayesian methods to address it are eminently applicable to the technological change problem. We next describe the formulation of this problem - as represented by the G-M optimization model - in a non-Bayesian framework, analogous to the Brock et al. macroeconomic analysis. Intuitively, we interpret the G-M framework as defining a class or family of "candidate" models parameterized by $\kappa$, but now the different members of this model set, and their policy implications, are not to be just considered by a decision-maker, but rather used explicitly in a precisely defined way.

\subsection{Min-max}

\footnotetext{
${ }^{4}$ Pizer and Popp [33] discuss both the theoretical and the empirical challenges in modeling energy-related technical change.
} 
Applied to model uncertainty, the min-max criterion, introduced by Wald [36], bases the decision upon the "worst-case" model - in the present context, the model associated with the highest cost. For notational brevity, define

$$
\Omega\left(A_{t}, H_{t}, S_{t}\right) \equiv C\left(A_{t}, H_{t}\right)+D\left(S_{t}\right) .
$$

A min-max framing of the ITC problem using the G-M model is

$$
\begin{aligned}
& \min _{A_{t}}\left\{\max _{\kappa} \int_{0}^{\infty} \Omega\left(A_{t}, H_{t}, S_{t}\right) e^{-\rho t} d t\right\} \\
& \text { s.t. } \\
& \frac{d}{d t} H_{t}=\alpha H_{t}+\kappa \Psi\left(H_{t}, A_{t}\right) \\
& \frac{d}{d t} S_{t}=-\varepsilon S_{t}+E_{t}^{0}-A_{t},
\end{aligned}
$$

subject to initial conditions and non-negativity constraints.

Heuristically, we interpret this problem and characterize its solution as follows. Assume that the support of $\kappa$ is a closed interval, $\kappa \in[0,1]$. Then note that abatement trajectory $\left\{A_{t}\right\}$ that is admissible for one value of $\kappa$ in this interval will be admissible for all values. Now, given admissible $\left\{A_{t}\right\}$, as $\kappa$ ranges over its domain the path of $H_{t}$ also varies, and therefore the timepath of values of $\Omega\left(A_{t}, H_{t}, S_{t}\right)$ varies as well. So, the discounted cost is maximized over this set, as a function of $\kappa$ (continuing to hold the $A_{t}$ trajectory fixed in the space of trajectories). This maximization yields a function of $\left\{A_{t}\right\}$, which is then minimized over all admissible such pairs. Regarding the solution, note first that because the discounted cost decreases in $\kappa$, the "inner" maximum is attained at $\kappa=0$. Then the "outer" problem is simply the cost minimization with autonomous technical change. Thus, the solution to the min-max problem corresponds to the "worst" case, in which no cost reduction is available from ITC.

\subsection{Min-max regret}

The idea of the min-max regret (MMR) criterion is to ameliorate the conservatism of the minmax criterion's dependence upon the worst case. The use of this criterion in energy modeling was pioneered by Loulou and Kanudia [37] in a regional-scale linear programming (LP) model. There has recently been an increase of work on this topic. Li et al. [38], Dong et al. [39], and Dong et al. [40] apply the regret criterion in different variations of LP power system modeling. Iverson [41] applies MMR in a version of Nordhaus's well-known "DICE" integrated assessment model, while Hall et al. [42] compare the results of MMR with an "info-gap" analysis as approaches to robustness analysis in DICE. Anthoff and Tol [43] analyze several decision criteria including MMR using the "FUND" integrated assessment model. 
In the present context, for a given policy - in our case, a trajectory $\left\{A_{t}\right\}$ - the "regret" associated with a model - defined by a value of $\kappa$ - is the difference in discounted cost between that associated with $\left\{A_{t}\right\}$ and the cost of the optimal policy for that model. For a given $\kappa$, let $A_{t}^{*}, H_{t}^{*}, S_{t}^{*}$ be the cost-minimizing control and state variables subject to the constraints listed in equations (4), and define

$$
M^{*}(\kappa) \equiv \int_{0}^{\infty} \Omega\left(A_{t}^{*}, H_{t}^{*}, S_{t}^{*}\right) e^{-\rho t} d t
$$

For given $A_{t}$ and $\kappa$, we can re-write the "regret" more succinctly as

$$
R\left(A_{t}, \kappa\right) \equiv \int_{0}^{\infty} \Omega\left(A_{t}, H_{t}, S_{t}\right) e^{-\rho t} d t-M^{*}(\kappa) .
$$

Then the overall min-max regret problem is

$$
\begin{aligned}
& \min _{A_{t}} \max _{\kappa} R\left(A_{t}, \kappa\right) \\
& \text { s.t. } \\
& \frac{d}{d t} H_{t}=\alpha H_{t}+\kappa \Psi\left(H_{t}, S_{t}\right) \\
& \frac{d}{d t} S_{t}=-\varepsilon S_{t}+E_{t}^{0}-A_{t} .
\end{aligned}
$$

\subsection{Expected cost minimization}

We noted above the complications involved in solving the model using expected cost minimization. However, it will be instructive to compare the non-Bayesian approach to model uncertainty represented by min-max regret solutions with the results of standard expected cost minimization applied to this model. In such a formulation, we assume that the uncertainty in $\kappa$ is captured by a probability distribution, and the model described by equations (4) above becomes

$$
\begin{aligned}
& \min _{A_{t}} \mathrm{E}_{\kappa} \int_{0}^{\infty} \Omega\left(A_{t}, H_{t}, S_{t}\right) e^{-\rho t} d t \\
& \text { s.t. } \\
& \frac{d}{d t} H_{t}=\alpha H_{t}+\kappa \Psi\left(H_{t}, A_{t}\right) \forall \kappa \in[0,1] \\
& \frac{d}{d t} S_{t}=-\varepsilon S_{t}+E_{t}^{0}-A_{t} \\
& H_{0}, S_{0} \text { given } \\
& A_{t}, H_{t}, S_{t} \geq 0,
\end{aligned}
$$

where $\mathbf{E}$ is the expectation operator. In the following sections we will implement this solution concept using, in turn, two different distributions for $\kappa$, to explore the dependence of the result on this assumption. This exercise is loosely related to the concept of "ambiguity aversion," 
which refers to a decision maker's model incorporating probabilistic uncertainty regarding one or more parameters, but the decision maker being uncertain as to which of several candidate distributions is the correct one to assign to these inputs (Schmeidler [44], Gilboa and Schmeidler [45]). A theory of decision-making under ambiguity has been developed to analyze the positive and normative implications of this type of model uncertainty.

\subsection{Theory and computation}

Notwithstanding this model's simplicity, analyzing MMR solutions is a problem for which computation is essential. The analysis of min-max solutions to optimal control problems has a long history; for example, Salmon [46] and Papageorgiou and Yannakakis [47] are early, and more recent, examples, respectively. The min-max regret problem in an optimal control setting, however, has not been the focus of research. The literatures on sensitivity analysis and suboptimality in optimal control dating back to the 1960s contain insights and techniques that are relevant to this problem. For example, Rekasius [48] and Durbeck [49] proposed Lyapunov-type techniques for estimating bounds on sub-optimal solutions using the optimal-value function, anticipating more recent work in the area of "dissipation inequalities." Witsenhausen [50] is an interesting attempt to develop a general theory relating uncertainty and sub-optimality. These two topics were also jointly analyzed by Wierzbicki [51], in another general theoretical treatment, as well as by Ronge [52]. Nevertheless, theoretical tools directly applicable to the optimal control MMR problem are not available. Thus, computational methods provide the means for obtaining and understanding MMR solutions in the present case. This illustrates the important fact that such methods can be highly useful for gaining insight even in the context of relatively simple models.

\section{Computational model forms}

\subsection{Functional forms}

To implement and solve the G-M model numerically, we switch to discrete time using the following functional forms and taking parameter values from Goulder and Mathai [12].

Abatement cost:

$$
C\left(A_{t}, H_{t}\right)=M_{C} \frac{A_{t}^{\alpha_{C_{1}}}}{\left(E_{t}^{0}-A_{t}\right)^{\alpha_{C_{2}}}} \frac{1}{H_{t}},
$$

with $M_{C}=83, \alpha_{C_{1}}=3, \alpha_{C_{2}}=2$. In addition, as described in Section 7 below, we analyze several cases with $H_{t}^{4}$ in the denominator.

Damage function:

$$
D\left(S_{t}\right)=M_{D} S^{\alpha_{D}}
$$

where $M_{D}=0.0012$ and $\alpha_{D}=2$. 


\section{Technological change - knowledge function:}

The knowledge production function is

$$
\Psi\left(I_{t}, H_{t}\right)=M_{\Psi} I_{t}^{\gamma} H_{t}^{\phi},
$$

where $\gamma=0.5, \phi=0.5$, and $H_{0}=1$. As described in Section 7 below, we first set $M_{\Psi}=0.0022$, but then also analyze cases with $M_{\Psi}=0.022$ and $M_{\Psi}=0.22$.

Technological change-dynamics:

The rate of autonomous change is assumed to be $0.5 \%$ per annum, i.e., $\alpha=0.005$.

$$
H_{t+1}=(1+\alpha) H_{t}+\kappa M_{\psi} H_{t}^{\phi} A_{t}^{\gamma} \text {. }
$$

Carbon stock dynamics:

$$
S_{t+1}=S_{t}+\beta\left(E_{t}^{0}-A_{t}\right)-\delta\left(S_{t}-S_{P R E}\right)
$$

where $\beta=0.64, \delta=0.008, S_{0}=360$, and the pre-industrial concentration is $S_{P R E}=278$ partsper-million by volume (ppmv).

The baseline emissions abatement scenario is "Representative Concentration Pathway (RCP) 8.5," generated using the intermediate-complexity climate model MAGICC 6 (Meinshausen et al. [53], [54]).

\subsection{Min-max regret}

We first note that in the discrete-time formulation, the min-max model becomes

$$
\begin{aligned}
& \min _{A_{t}} \max _{\kappa} \sum_{t=0}^{\infty} \Omega\left(A_{t}, H_{t}, S_{t}\right) e^{-\rho t} \\
& \text { s.t. } \\
& H_{t+1}=(1+\alpha) H_{t}+\kappa M_{\psi} H_{t}^{\phi} A_{t}^{\gamma} \\
& S_{t+1}=S_{t}+\beta\left(E_{t}^{0}-A_{t}\right)-\delta\left(S_{t}-S_{P R E}\right) \\
& A_{t}, H_{t}, S_{t} \geq 0 .
\end{aligned}
$$

As in the theoretical, continuous-time version of this problem, the solution is immediately seen to be at $\kappa=0$, because the discounted cost declines monotonically as $\kappa$ increases, for any given abatement path $A_{t}$. We therefore turn our attention to the min-max regret problem. To formulate a computational version, we first define

$$
f(\kappa) \equiv \min _{A_{t}}\left(\sum_{t=0}^{\infty} \Omega\left(A_{t}, H_{t}(\kappa), S_{t}\right) e^{-\rho t}\right) .
$$

Then the regret function is 


$$
R(A, H(\kappa), S, \kappa) \equiv\left(\sum_{t=0}^{\infty} \Omega\left(A_{t}, H_{t}(\kappa), S_{t}\right) e^{-\rho t}\right)-f(\kappa)
$$

where $A, H(\kappa)$ and $S$ respectively represent the paths of $A_{t}, H_{t}(\kappa)$ and $S_{t}$ over time, and the discrete-time min-max regret model is

$$
\begin{aligned}
& \min _{A_{t}} \max _{\kappa} R(A, H(\kappa), S, \kappa) \\
& \text { s.t. } \\
& H_{t+1}=(1+\alpha) H_{t}+\kappa M_{\psi} H_{t}^{\phi} A_{t}^{\gamma} \\
& S_{t+1}=S_{t}+\beta\left(E_{t}^{0}-A_{t}\right)-\delta\left(S_{t}-S_{P R E}\right) \\
& A_{t}, H_{t}, S_{t} \geq 0 .
\end{aligned}
$$

For computational implementation, we discretize $\kappa$ over the unit interval, with values $\kappa_{1} \ldots, \kappa_{n}$, so that the objective becomes

$$
\min _{A_{t}} \max _{\kappa \in\left\{\kappa_{1}, \ldots, \kappa_{n}\right\}} R(A, H(\kappa), S, \kappa) .
$$

Next, we transform this to a more standard optimization form as follows:

$$
\begin{aligned}
& \min _{A_{t}} \lambda \\
& \text { s.t. } \\
& \lambda \geq R\left(A, H^{(i)}, S, \kappa_{i}\right), i=1, \ldots, n \\
& H_{t+1, i}=(1+\alpha) H_{t, i}+\kappa_{i} M_{\psi} H_{t, i}^{\phi} A_{t}^{\gamma}, i=1, \ldots, n \\
& S_{t+1}=S_{t}+\beta\left(E_{t}^{0}-A_{t}\right)-\delta\left(S_{t}-S_{P R E}\right) \\
& A_{t}, H_{t, i}, S_{t} \geq 0,
\end{aligned}
$$

where $H^{(i)}$ represents the path of knowledge with the given $\kappa_{i}$, i.e., $\left\{H_{t, i}: t=0,1, \ldots\right\}$.

Numerically we replace the infinite-horizon problem by a finite but large horizon one. For example, in our numerical implementation in Section 7 below, we use 490 years as the horizon because with discounting the present value of costs incurred after 490 years is negligible..

\subsection{Expected cost minimization}

One key difference between the expected cost minimization model and the min-max regret model is that the former has to assume a prior distribution over the uncertain parameters while the latter assumes no prior distribution. Adopting our notation to reflect the dependence of $H_{t}$ on $\kappa$, a discrete-time formulation of this version of the expected cost minimization model is 


$$
\begin{aligned}
& \min _{A_{t}} \mathrm{E}_{\kappa} \sum_{t=0}^{\infty}\left(\Omega\left(A_{t}, H_{t}(\kappa), S_{t}\right)\right) e^{-\rho t} \\
& \text { s.t. } \\
& H_{t+1}(\kappa)=(1+\alpha) H_{t}(\kappa)+\kappa M_{\psi} H_{t}(\kappa)^{\phi} A_{t}^{\gamma} \forall \kappa \in[0,1] \\
& S_{t+1}=S_{t}+\beta\left(E_{t}^{0}-A_{t}\right)-\delta\left(S_{t}-S_{P R E}\right) \\
& A_{t}, H_{t}, S_{t} \geq 0 .
\end{aligned}
$$

Assuming that the distribution of $\kappa$ is continuous, the expectation in equation (22) becomes an integration and then can be approximated using a quadrature rule, so we transform the model (22) to:

$$
\begin{aligned}
& \min _{A_{t}} \sum_{i=1}^{n} w_{i}\left(\sum_{t=0}^{\infty} \Omega\left(A_{t}, H_{t, i}, S_{t}\right) e^{-\rho t}\right) \\
& \text { s.t. } \\
& H_{t+1, i}=(1+\alpha) H_{t, i}+\kappa_{i} M_{\psi} H_{t, i}^{\phi} A_{t}^{\gamma}, i=1, \ldots, n \\
& S_{t+1}=S_{t}+\beta\left(E_{t}^{0}-A_{t}\right)-\delta\left(S_{t}-S_{P R E}\right) \\
& A_{t}, H_{t, i}, S_{t} \geq 0,
\end{aligned}
$$

where $\kappa_{i}$ are quadrature nodes and $w_{i}$ are quadrature weights. The best choice for the quadrature nodes and weights depends on the integrand function and the prior distribution. (See Judd [55] and Cai [56] for detailed discussions of numerical integration, including the issues discussed here.) For example, the Gaussian-Hermite quadrature rule is used for an expectation with normal distribution. Since $\kappa$ is bounded in $[0,1]$, we choose the composite Simpson's rule with an approximation error of order $h^{4}$ for smooth integrand functions, while the traditional composite midpoint rule has an error of order $h^{2}$, where $h$ is the step size of quadrature nodes. As in the min-max regret case, in the computation of expected cost we approximate the infinitehorizon sum in the objective of the equation (23) by a finite-horizon sum because, due to discounting, cost terms in the long run have virtually no effect on the optimal solution.

Monte Carlo methods could also be used for approximating the expectation in the objective of equation (22), and their transformed models have the same form of (23), while $\kappa_{i}$ are simulated nodes and $w_{i}$ are $1 / n$, and it has about $n T$ constraints where $T$ is the finite horizon for approximating the infinite horizon problem. We know that Monte Carlo methods will have only $1 / \sqrt{n}$ accuracy. This implies that if we want to have $O\left(10^{-6}\right)$ accuracy, we will need about $10^{12} T$ constraints that make it infeasible to be solved. However, the composite Simpson's rule has much higher accuracy $O\left(M h^{4}\right)$ where $h=1 /(n-1)$ and $M$ is a constant depending on the smoothness of the integrand function, so usually it will have $O\left(10^{-6}\right)$ accuracy by letting 
$n=101$, and then the model (23) has about $101 T$ constraints and can be easily solved with a modern optimization solver.

As presented in Section 7 below, we implemented the expected cost minimization model in two ways:

- Uniform distribution: We assumed that $\kappa$ is uniform and continuous on [0,1]. The quadrature nodes $\kappa_{i}$ are equally spaced in $[0,1]$ with an odd number of nodes, $n$. The quadrature weights are given by the Simpsons' rule: $w_{1}=w_{n}=h / 3$, $w_{2}=w_{4}=\ldots=w_{n-1}=4 h / 3$, and $w_{3}=w_{5}=\ldots=w_{n-2}=2 h / 3$.

- Beta distribution: We assumed that the prior distribution of $\kappa$ is Beta, with density

$$
f(x ; \alpha, \beta)=\frac{1}{B(\alpha, \beta)} x^{\alpha-1}(1-x)^{\beta-1}, x \in[0,1],
$$

where $\alpha>0$ and $\beta>0$ are shape parameters and $B(\alpha, \beta)$ is the Beta function,

$$
B(\alpha, \beta)=\frac{(\alpha-1) !(\beta-1) !}{(\alpha+\beta-1) !}
$$

In this case, the mean of $\kappa$ is $1 /(1+\beta / \alpha)$. The quadrature nodes $\kappa_{i}$ are equally spaced in $[0,1]$ with an odd number of nodes, $n$. The quadrature weights are derived from the Simpsons' rule: $w_{1}=f\left(\kappa_{1} ; \alpha, \beta\right) h / 3, w_{n}=f\left(\kappa_{n} ; \alpha, \beta\right) h / 3, w_{2 i}=4 f\left(\kappa_{2 i} ; \alpha, \beta\right) h / 3$ for $i=1, \ldots,(n-1) / 2$, and $w_{2 i+1}=2 f\left(\kappa_{2 i+1} ; \alpha, \beta\right) h / 3$ for $i=1, \ldots,(n-3) / 2$.

When we choose a large number of quadrature nodes, the model (23) could become too large to be solved. Even if we choose a smaller number of nodes, obtaining a solution could still be quite time consuming for problems with many state variables. (In this paper, we choose $n=$ 101 and the calculations are fast because the model has only two state variables). The following deterministic model, in which $\kappa$ is assigned its mean value $\bar{\kappa}$, is an example of an often-used alternative way to estimate the solution. We note, however, that our numerical results show that the resulting approximation to the solution of the expected cost minimization problem may be very coarse in this case:

$$
\begin{aligned}
& \min _{A_{t}} \sum_{t=0}^{\infty}\left(\Omega\left(A_{t}, H_{t}, S_{t}\right)\right) e^{-\rho t} \\
& \text { s.t. } \\
& H_{t+1}=(1+\alpha) H_{t}+\bar{\kappa} M_{\psi} H_{t}^{\phi} A_{t}^{\gamma} \\
& S_{t+1}=S_{t}+\beta\left(E_{t}^{0}-A_{t}\right)-\delta\left(S_{t}-S_{P R E}\right) \\
& A_{t}, H_{t}, S_{t} \geq 0 .
\end{aligned}
$$

Although this model (26) may provide a less-accurate solution than model (23) (i.e., with respect to the underlying model's true solution), it is also the case that solving model (26) is much faster than solving model (23), because (26) has only $O(T)$ constraints while (23) has $O(n T)$ 
constraints, where $T$ is the finite horizon for approximating the infinite horizon problem, and $n$ is the number of quadrature nodes used in (23). Thus, the use of model (26) can still be warranted on computational tractability grounds as well as for the insight into underlying model structure that may be gained by using and comparing the results of both solution strategies. This is illustrated in our results in the following section.

\section{Numerical results}

\subsection{Two Uncertain Parameters}

For all of our computational modeling, we use annual time steps from year 2010 to year 2500. We recall that the baseline emissions abatement scenario is "Representative Concentration Pathway (RCP) 8.5" (Meinshausen et al. [53], [54]). We use CONOPT [57] in the GAMS [58] environment to solve the models. For the model (23), we use the composite Simpson's rule with $n=101$ equally-spaced quadrature nodes over [0,1], i.e., $\kappa_{i}=(i-1) h$ for $i=1, \ldots, 101$, with $h=1 /(n-1)$. We also note that discretization of $\kappa$ over the unit interval allows us to extend the results of G-M, who analyzed the two values $\kappa=0$ and $\kappa=1$.

In all our numerical results, there is a "turning point" - a maximum abatement level - at year 2150. This is a consequence of our use of the Representative Concentration Pathway 8.5 emissions scenario, which embodies this specific maximum.

Figure 1 presents the basic comparison of abatement paths in the solutions of the G-M model with autonomous and induced technical change, respectively, and with the min-max regret criterion. It shows that the latter decision rule in a sense "balances" between the two polar technical change assumptions. 
Figure 1. Abatement paths in computational G-M model with induced and autonomous technical change (minimum cost), and with min-max regret criterion

\section{Abatement when $\mathrm{M}_{\psi}=0.0022$}

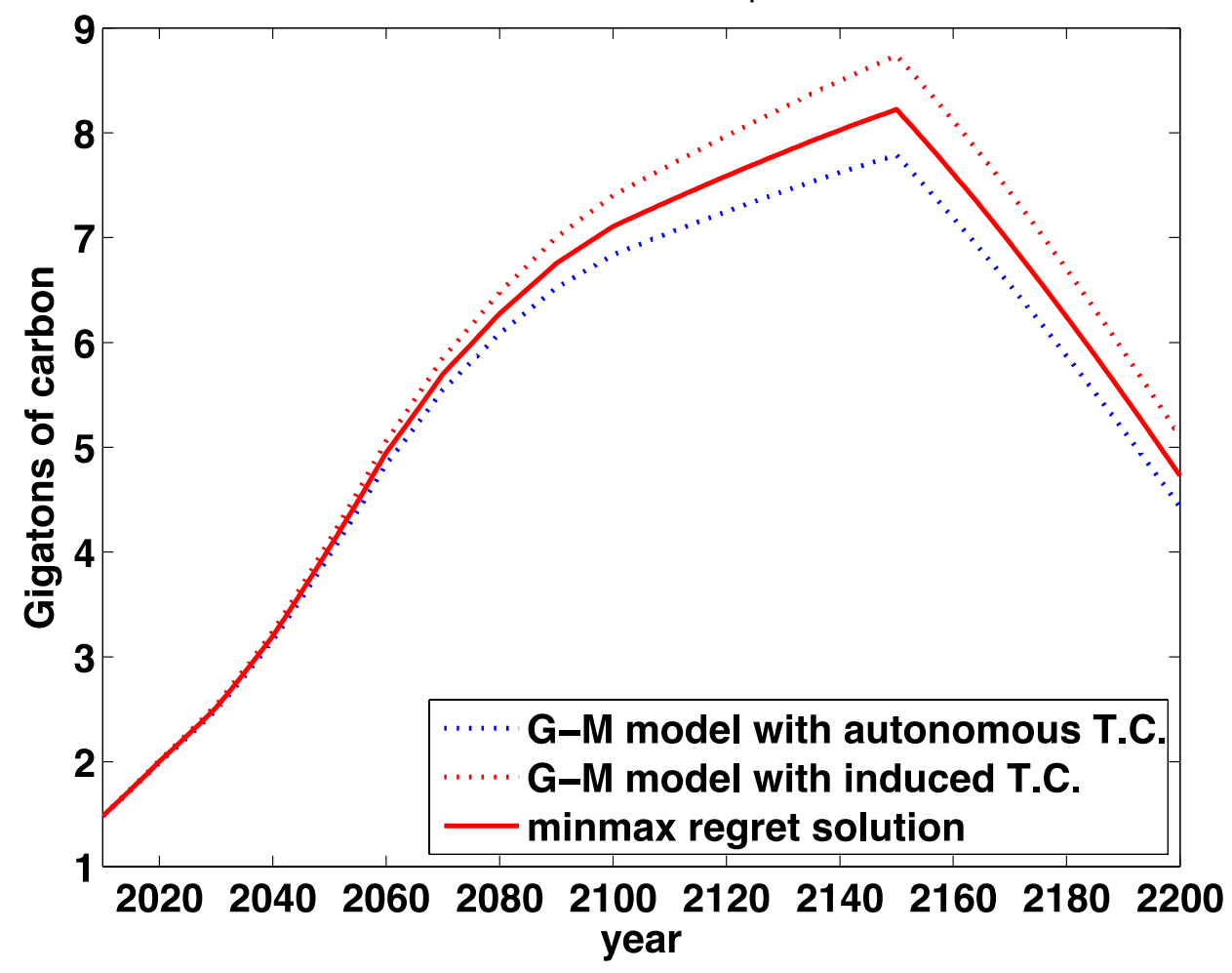

This initial comparison raises several questions. Is the approximate symmetry of the outcome - i.e., with the min-max regret solution roughly "midway" between the two standard solutions - a consequence of the decision rule, the particular characteristics of the model, or some combination? How is the "spread" of abatement paths - that is, the difference between the induced and the autonomous cases, respectively - related to our assumptions? Finally, per our discussion above, how does the non-Bayesian solution compare with a conventional expected cost-minimization solution?

In our next set of results, we expand our set of comparisons in two ways to address these issues. First, we solve the model for additional specifications of the knowledge production function - specifically, recalling that

$$
H_{t+1}=(1+\alpha) H_{t}+\kappa M_{\psi} H_{t}^{\phi} A_{t}^{\gamma}
$$

and that $M_{\Psi}=0.0022$ in the results displayed in Figure 1, we also solve for $M_{\Psi}=0.022$ and $M_{\Psi}=0.22$. For any magnitude of $\kappa>0$, these higher values imply a great relative contribution of induced technical change relative to autonomous in determining the level of the knowledge stock $H_{t}$ in each period. The induced, autonomous, and min-max regret solutions are computed for each of these three values. 
Second, we also now solve the expected cost minimization version of the model assuming a uniform distribution for $\kappa$ as described in Section 6.3. For this case also, we solve for the three values of $M_{\Psi}$, as well as for the model with $\kappa$ fixed at its mean value.

The results are shown in Figure 2. In the first panel, we see that the min-max regret, expected cost, and minimum cost with $\kappa$ set to its mean value essentially coincide exactly. The second and third panels show that increasing the value of $M_{\Psi}$ not only results in substantial divergence of abatement paths overall, but also an overall increase in their average magnitude. Moreover, it is interesting to note that the min-max regret solution with higher values of $M_{\Psi}$ becomes relatively "conservative" - i.e., lower in magnitude than all of the other cases except autonomous technical change. 
Figure 2. Abatement paths in computational G-M model - varying $M_{\psi}$ with induced and autonomous technical change (minimum cost), min-max regret criterion, and expected minimum cost with uniform prior on $\kappa$
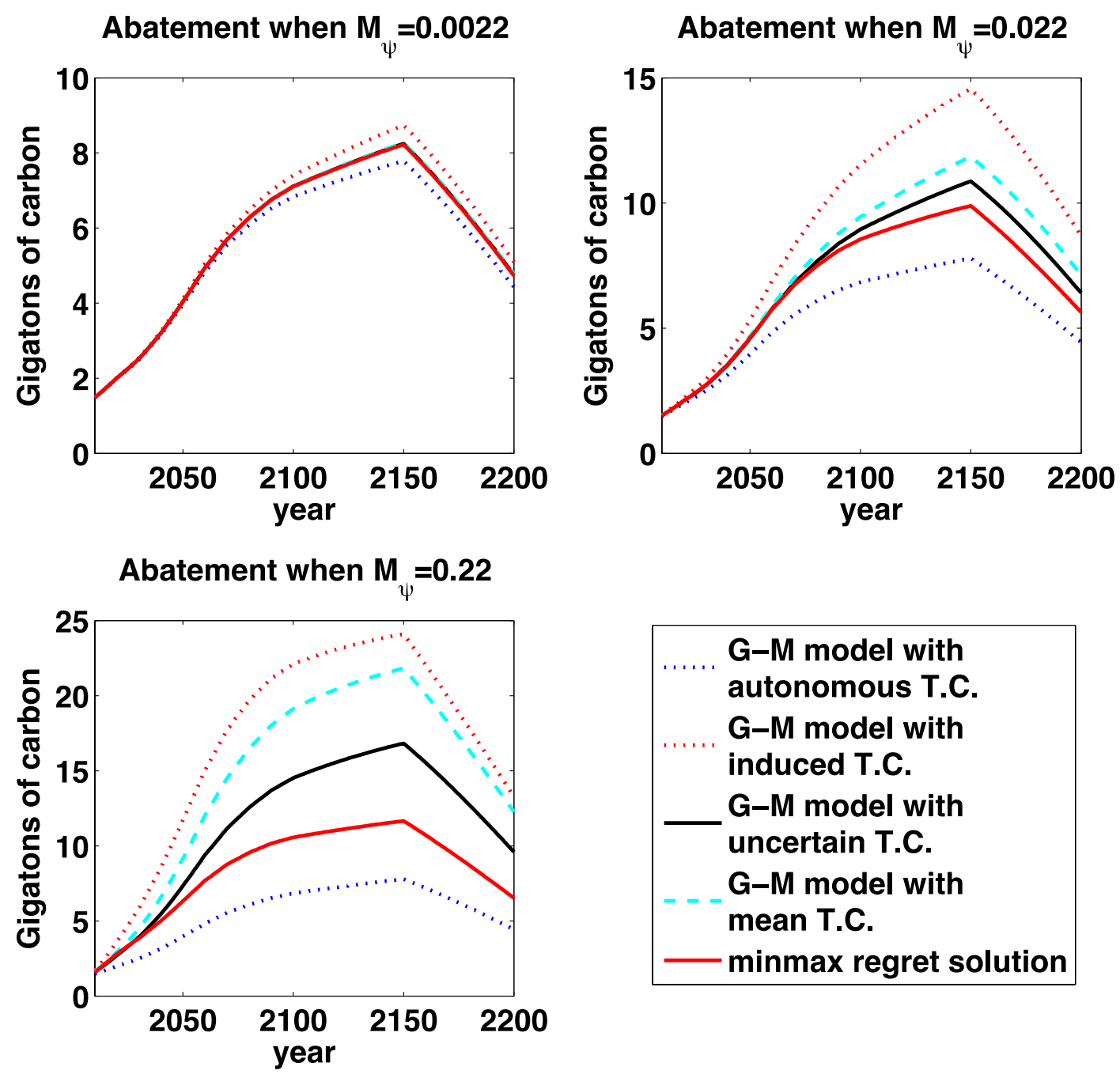

In our next set of computations we explore the effect of changing the prior distribution on $\kappa$, from uniform to Beta (as described in Section 6), with two different combinations of values for the shape parameters. The results are shown in Figure 3. Overall, the magnitudes of the abatement paths are comparable to those shown in Figure 2 with a uniform prior. It is interesting to note that in the $M_{\Psi}=0.022$ case, the min-max regret solution exceeds both the optimal (minimum expected cost) and mean solutions with Beta $(1,3)$ until the maximum point of each, then crosses below the mean, but not the optimal, solution. 
Figure 3. Abatement paths in computational G-M model - linear $\boldsymbol{H}_{\boldsymbol{t}}$ in cost function, and varying $M_{\psi}$ with induced and autonomous technical change (minimum cost), min-max regret criterion, and expected minimum cost with two instances of Beta prior on $\boldsymbol{\kappa}$
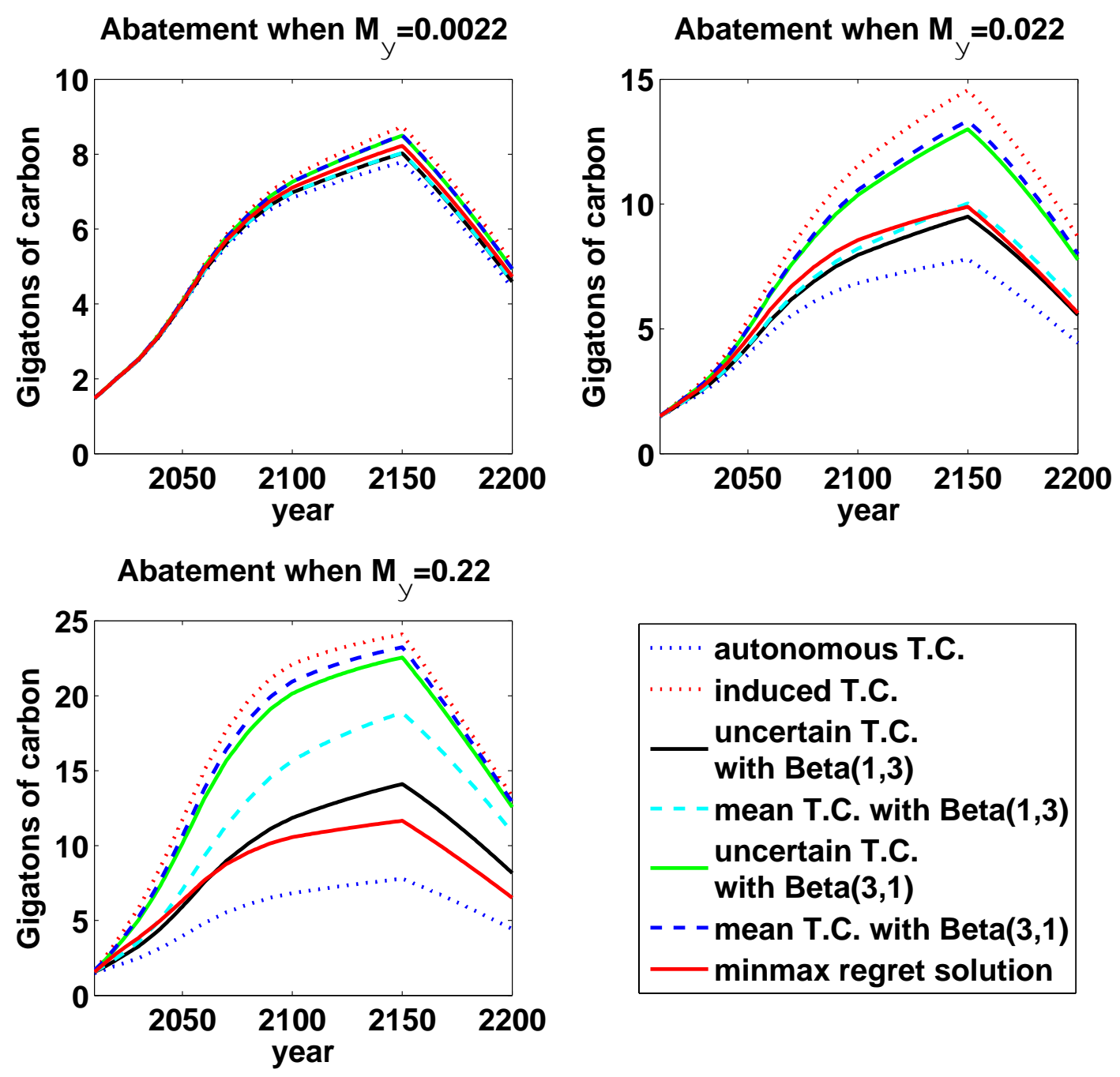

To further explore the interactions between the structure of the model and the solution concept, we next conduct the same set of computations but with increased curvature in the cost functionspecifically, with the knowledge stock $H_{t}$ raised to the $4^{\text {th }}$ (rather than the $1^{\text {st }}$ ) power:

$$
C\left(A_{t}, H_{t}\right)=M_{C} \frac{A_{t}^{\alpha_{C_{1}}}}{\left(E_{t}^{0}-A_{t}\right)^{\alpha_{C_{2}}}} \frac{1}{H_{t}^{4}} .
$$

The results are shown in Figure 4. Again we see a divergence among paths as $M_{\Psi}$ increases, as well as an even greater increase in the overall magnitudes of abatement - that is, increasing the curvature of the cost function raises not only min-max regret abatement, but also optimal abatement in every case. It is also interesting to note that the abatement paths for induced and mean technical change, respectively, are essentially the same for the highest value of $M_{\Psi}$. 
Figure 4. Abatement paths in computational G-M model - "quartic" $H_{t}$ in cost function, and varying $M_{\psi}$, with induced and autonomous technical change (minimum cost), minmax regret criterion, and expected minimum cost with uniform prior on $\kappa$
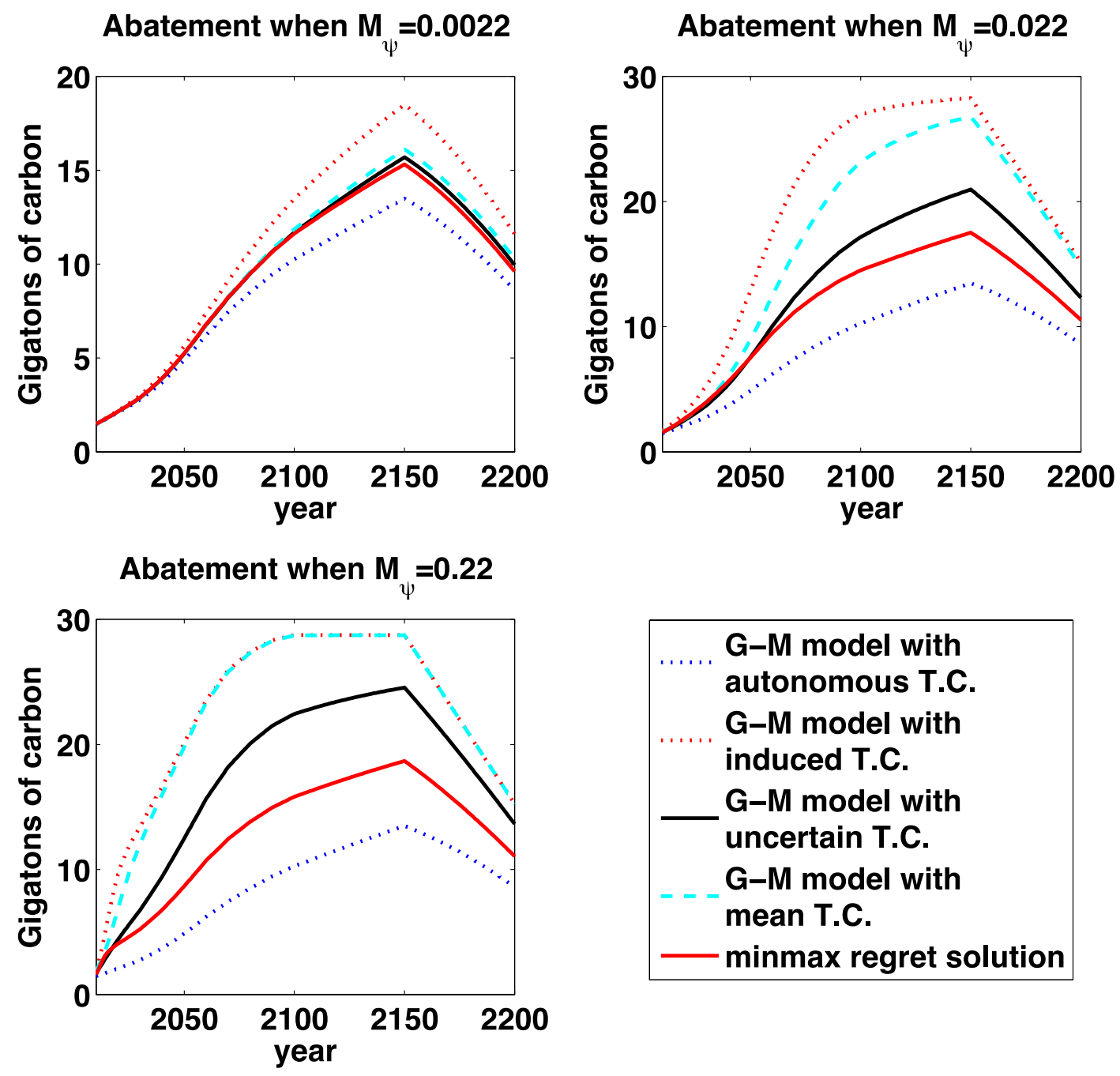

\subsection{Multiple Uncertain Parameters}

In the previous subsection, we conducted sensitivity analysis over two parameters: $M_{\Psi}$ and the exponent of the power function of the knowledge stock $H_{t}$ in the cost function, denoted $d$. In the analysis, we computed solutions of various methods including the min-max regret method for $\operatorname{each} M_{\Psi} \in\{0.0022,0.022,0.22\}$ and $d \in\{1,4\}$. 
In applying the min-max regret approach, we assume that the hypothetical decision maker knows neither the distribution of the uncertain parameter $\kappa$ nor its mean, while the other methods assume that the decision maker knows the distribution or its mean. If we assume that $M_{\Psi}$ and $d$ are also uncertain, and the decision maker does not know their probability distributions (for simplicity, we assume that $M_{\Psi} \in\{0.0022,0.022,0.22\}$ and $\left.d \in\{1,4\}\right)$, we then extend the previous min-max regret analysis over one uncertain parameter to solve the problem with multiple uncertain parameters. Now, the abatement cost function is also dependent on $d$, i.e.,

$$
C\left(A_{t}, H_{t}, d\right)=M_{C} \frac{A_{t}^{\alpha_{C_{1}}}}{\left(E_{t}^{0}-A_{t}\right)^{\alpha_{C_{2}}}} \frac{1}{H_{t}^{d}},
$$

and we let $\Omega\left(A_{t}, H_{t}, S_{t}, d\right) \equiv C\left(A_{t}, H_{t}, d\right)+D\left(S_{t}\right)$, where the transition law of $H_{t}$ depends on $\kappa$ and $M_{\Psi}$. Similarly, we let

$$
f\left(\kappa, M_{\psi}, d\right) \equiv \min _{A_{t}}\left(\sum_{t=0}^{\infty} \Omega\left(A_{t}, H_{t}\left(\kappa, M_{\psi}\right), S_{t}, d\right) e^{-\rho t}\right),
$$

so that the regret function is

$$
R\left(A, H\left(\kappa, M_{\psi}\right), S, \kappa, d\right) \equiv\left(\sum_{t=0}^{\infty} \Omega\left(A_{t}, H_{t}\left(\kappa, M_{\psi}\right), S_{t}, d\right) e^{-\rho t}\right)-f\left(\kappa, M_{\psi}, d\right) .
$$

Thus, our min-max regret model becomes

$$
\begin{aligned}
& \min _{A_{t}} \lambda \\
& \text { s.t. } \\
& \lambda \geq R\left(A, H^{(i, j)}, S, \kappa_{i}, M_{\psi, j}, d\right), i=1, \ldots, n, \quad j=1,2,3, \quad d=1,4 \\
& H_{t+1, i, j}=(1+\alpha) H_{t, i, j}+\kappa_{i} M_{\psi, j} H_{t, i, j}^{\phi} A_{t}^{\gamma}, i=1, \ldots, n, \quad j=1,2,3 \\
& S_{t+1}=S_{t}+\beta\left(E_{t}^{0}-A_{t}\right)-\delta\left(S_{t}-S_{P R E}\right) \\
& A_{t}, H_{t, i, j}, S_{t} \geq 0,
\end{aligned}
$$

where $H^{(i, j)}$ represents the path of knowledge with the given $\kappa_{i}$ and $M_{\Psi, j}$, i.e.,

$$
\left\{H_{t, i, j}: t=0,1, \ldots\right\} \text {. }
$$

Figure 5 shows the solution of the min-max regret method over these three uncertain parameters (the red solid line), and compares it with the solution of the min-max regret method over only one uncertain parameter, $\kappa$, with various given values of $M_{\Psi}$ and $d$. The results show that the sensitivity analysis over $M_{\Psi}$ and $d$ cannot give one robust policy for decision makers, while the min-max regret solution with these three multiple uncertain parameters does. 
Figure 5. Abatement paths in computational G-M model - min-max regret criterion with three uncertain parameters $\left(\kappa, M_{\psi}, d\right)$, and sensitivity analysis of min-max regret criterion with only one uncertain parameter $\boldsymbol{\kappa}$

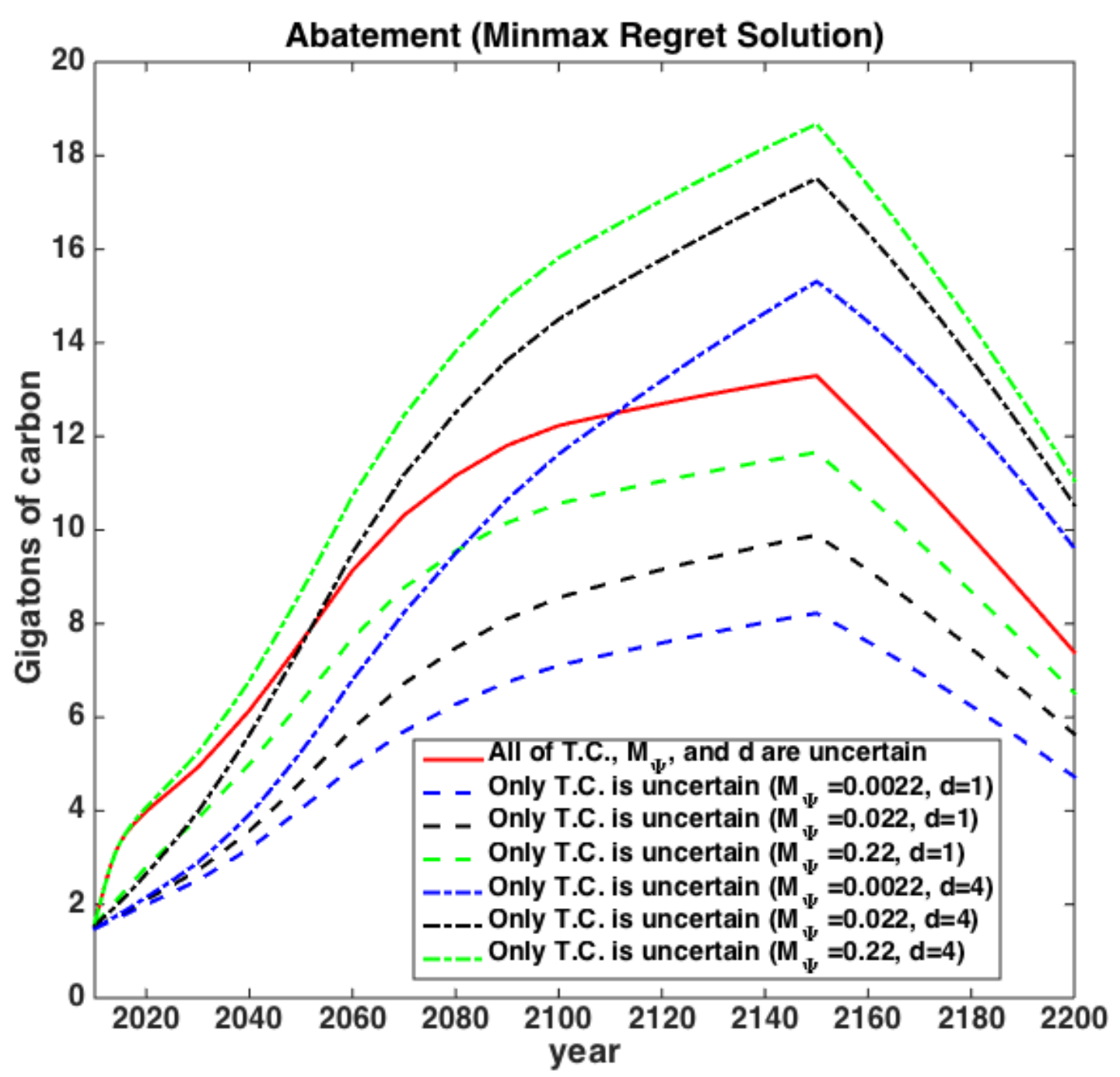

\section{Conclusion}

This paper has focused on fundamental model uncertainty in the study of $\mathrm{CO} 2$ emissions abatement from the energy system, has analyzed a non-Bayesian approach - the minmax regret decision rule - to address this uncertainty, and has compared the results with those obtained from expected cost minimization. Our findings demonstrate the insights available from using a nonBayesian, robust decision-making approach to the technological change problem; it shows that interpreting the technical change problem in terms of model uncertainty in principle enables a 
policy-maker to formally incorporate the assumptions of both autonomous and induced technical change in an integrated manner, without having to overcome the difficult problem of assigning numerical prior probabilities to these alternative representations.

As in many other fields of application, computational modeling has become an indispensable analytical methodology for energy and environmental policy. The development and widespread adoption of energy modeling over the past several decades accompanied by the attenuation of serious, sustained work on model validation, however, has arguably given rise to fundamental epistemological issues, with significant implications for model-based policy analysis, that have yet to be systematically addressed. The emergence of multiple models of implicitly equal validity, without formal guidance to policy-makers regarding their joint application, is an important example. If this state-of-affairs reflects the persistence of irreducible uncertainties in our understanding of the energy system and its relationship to the economy, then developing methods to enable policy makers to rationally deal with it is a high priority. The pioneering work of macroeconomists on model uncertainty provides a compelling starting point for such an effort. The work described in this paper is only a modest first step, but we hope to have demonstrated both the importance and the feasibility of bringing the macroeconomists' insights and techniques to bear on energy modeling.

\section{References}

[1] Fischer, Carolyn, and Richard D. Morgenstern. 2006. "Carbon Abatement Costs: Why the Wide Range of Estimates?” The Energy Journal 27 (2): 73-86.

[2] Hansen, Lars Peter, and Thomas J. Sargent. 2005. "Robust Estimation and Control under Commitment.” Journal of Economic Theory 124 (2): 258-301.

[3] Hansen, Lars Peter, and Thomas J. Sargent. 2007a. "Recursive Robust Estimation and Control without Commitment." Journal of Economic Theory 136: 1-27.

[4] Hansen, Lars Peter, and Thomas J. Sargent. 2007b. Robustness. Princeton: Princeton University Press.

[5] Brock, William A., and Steven N. Durlauf, Kenneth D. West. 2003. "Policy Evaluation in Uncertain Economic Environments.” Brookings Papers on Economic Activity I, 2003: 235-322.

[6] Brock, William A., and Steven N. Durlauf, James M. Nason, Giacomo Rondina. 2007a. "Simple versus optimal rules as guides to policy." Journal of Monetary Economics 54: 13721396.

[7] Brock, William A., and Steven N. Durlauf, Kenneth D. West. 2007b. "Model uncertainty and policy evaluation: Some theory and empirics." Journal of Econometrics 136: 629-664.

[8] Solow, Robert M. 1956. "A Contribution to the Theory of Economic Growth.” Quarterly Journal of Economics 70, February: 65-94.

[9] McInerney, David, and Robert Lempert, Klaus Keller. 2012. "What are robust strategies in the face of uncertain climate threshold responses?" Climatic Change 112: 547-568. 
[10] Hogan, William W. 1978. "Energy Modeling: Building Understanding for Better Use." Paper presented at the $2^{\text {nd }}$ Lawrence Symposium on The Systems and Decision Sciences, Berkeley, California, October 3.

[11] Peace, Janet, and John Weyant. 2008. "Insights not Numbers: The Appropriate Use of Economic Models." White paper, Pew Center on Global Climate Change, Washington, DC: April.

[12] Goulder, Lawrence H., and Koshy Mathai. 2000. "Optimal CO2 Abatement in the Presence of Induced Technological Change." Journal of Environmental Economics and Management 39, $1-28$.

[13] Murphy, Frederic H., and Susan H. Shaw. 1995. "The Evolution of Energy Modeling at the Federal Energy Administration and the Energy Information Administration." Interfaces 25 (5), Sept. - Oct.: 173-193.

[14] Greenberger, Martin, and Matthew A. Crenson, Brian L. Crissey. 1976. Models in the Policy Process: Public Decision Making in the Computer Era. New York: Russell Sage Foundation.

[15] Gruhl, J., and N. Gruhl. 1978. "Methods and Examples of Model Validation - An Annotated Bibliography.” MIT Energy Laboratory Working Paper MIT-EL 78-022WP, July.

[16] Greenberger, Martin, and Richard Richels. 1979. "Assessing Energy Policy Models: Current State and Future Directions.” Annual Review of Energy 4: 467-500.

[17] National Bureau of Standards (NBS). 1980. Validation and Assessment Issues on Energy Models: Proceedings of a Workshop held at the National Bureau of Standards, Gaithersburg, Maryland, January 10-11, 1979. Saul I. Gass, Ed. NBS Special Publication, U. S. Department of Commerce, Washington, DC, February.

[18] Huntington, Hillard G., and John P. Weyant, James L. Sweeney. 1982. "Modeling for Insights, not Numbers: the Experiences of the Energy Modeling Forum." OMEGA - The International Journal of Management Science 10 (5): 449-462.

[19] Dawkins, Christina, and T. N. Srinivasan, John Whalley. 2001. "Calibration." Chapter 58 in J. J. Heckman and E. Leamer, Eds., Handbook of Econometrics, Volume 5. Elsevier Science B. $\mathrm{V}$.

[20] Kann, Antje, and John P. Weyant. 2000. "Approaches for performing uncertainty analysis in large-scale energy/economic policy models." Environmental Modeling and Assessment 5: 29-46. [21] Clarke, L., and J. Edmonds, H. Jacoby, H. Pitcher, J. Reilly, R. Richels. 2007. Scenarios of Greenhouse Gas Emissions and Atmospheric Concentrations. Sub-report 2.1A of Synthesis and Assessment Product 2.1 by the U. S. Climate Change Science Program and the Subcommittee on Global Change Research. Department of Energy, Office of Biological \& Environmental Research, Washington, DC, USA, 154 pp. 
[22] Parker, Larry, and Brent Yacobucci. 2008. "Climate Change: Costs and Benefits of S. 2191.” CRS Report for Congress RL34489, Congressional Research Service, May 15.

[23] Hogan, William W., and Alan S. Manne. 1977. "Energy-Economy Interactions: The Fable of the Elephant and the Rabbit?" Appendix B in Energy and The Economy, Energy Modeling Forum (EMF) Report 1, Volumes 1 and 2, Stanford University, September.

[24] Swan, Trevor W. 1956. "Economic Growth and Capital Accumulation.” Economic Record 32, November: 334-361.

[25] Hogan, William W., and Dale W. Jorgenson. 1991. "Productivity Trends and the Cost of Reducing CO2 Emissions.” The Energy Journal 12 (1), Issue 1: 67-86.

[26] Romer, Paul M. 1986. “Increasing Returns and Long-Run Growth.” Journal of Political Economy 94 (5), October: 1002-1037.

[27] Romer, Paul M. 1990. “Endogenous Technological Change.” Journal of Political Economy 98 (5), Part 2: S71-S102.

[28] Lucas, Robert E., Jr. 1987. “On the Mechanics of Economic Development.” Journal of Monetary Economics 22: 3-42.

[29] Gillingham, Kenneth, and Richard G. Newell, William A. Pizer. 2008. "Modeling endogenous technological change for climate policy analysis." Energy Economics 30: 27342753.

[30] Buonanno, Paolo, and Carlo Carraro, Marzio Galeotti. 2003. "Endogenous induced technical change and the costs of Kyoto." Resource and Energy Economics 25: 11-34

[31] Popp, David. 2006. "Innovation in climate policy models: Implementing lessons from the economics of R\&D.” Energy Economics 28: 596-609.

[33] Pizer, William A., and David Popp. 2008. "Endogenizing technological change: Matching empirical evidence to modeling needs.” Energy Economics 30: 2754-2770.

[34] Popp, David. 2010. "Innovation and Climate Policy." Annual Review of Resource Economics Volume 2, 2010: 275-298.

[35] LaFrance, Jeffrey, and L. Dwayne Barney. 1991. "The envelope theorem in dynamic optimization.” Journal of Economic Dynamics and Control 15: 355-385.

[36] Wald, A. 1950. Statistical Decision Functions. New York: Wiley.

[37] Loulou, Richard, and Amit Kanudia. 1999. "Minimax regret strategies for greenhouse gas abatement: methodology and application." Operations Research Letters 25 (5), December: 219230.

[38] Li, Y. P., and G. H. Huang, X. Chen. 2011. “An interval-valued minimax-regret analysis approach for the identification of optimal greenhouse-gas abatement strategies under uncertainty." Energy Policy 39: 4313-4324. 
[39] Dong, C., and G. H. Huang, Y. P. Cai, Y. Xu. 2011. “An interval-parameter minimax regret programming approach for power management systems planning under uncertainty." Applied Energy 88: 2835-2845.

[40] Dong, C. J., and Y. P. Li, G. H. Huang. 2014. "Superiority-inferiority modeling coupled minimax-regret analysis for energy management systems." Applied Mathematical Modelling 38: 1271-1287.

[41] Iverson, Terrence. 2012. "Communicating Trade-offs amid Controversial Science: Decision Support for Climate Policy.” Ecological Economics 77: 74-90.

[42] Hall, Jim W., and Robert J. Lempert, Klaus Keller, Andrew Hackbarth, Christophe Mijere, David J. McInerney. 2012. "Robust Climate Policies Under Uncertainty: A Comparison of Robust Decision-Making and Info-Gap Methods.” Risk Analysis 32 (10): 1657-1672.

[43] Anthoff, David, and Richard S. J. Tol. 2013. "Climate policy under fat-tailed risk: an application of FUND.” Annals of Operations Research DOI 10.1007/s10479-013-1343-2.

[44] Schmeidler, David. 1989. "Subjective Probability and Expected Utility Without Additivity." Econometrica 57 (3): 571-587.

[45] Gilboa, Itzhak, and David Schmeidler. 1989. "Maxmin expected utility with non-unique prior.” Journal of Mathematical Economics 18 (2): 141-153.

[46] Salmon, D. M. 1968. "Minimax Controller Design.” IEEE Transactions on Automatic Control, Vol. AC-13, No. 4, August: 369-376.

[47] Papageorgiou, Nikolaos S., and Nikolaos Yannakakis. 2002. "Minimax control of nonlinear evolution equations." Applied Mathematics and Computation 131: 271-297.

[48] Rekasius, Z. V. 1964. "Suboptimal Design of Intentionally Nonlinear Controllers." IEEE Transactions on Automatic Control, October: 380386.

[49] Durbeck, Robert C. 1965. "An Approximation Technique for Suboptimal Control.” IEEE Transactions on Automatic Control Vol. AC-10, no. 2, April: 144-149.

[50] Witsenhausen, H. S. 1970. “On Performance Bounds for Uncertain Systems.” SIAM Journal of Control Vol. 8 No. 1, February: 55-89.

[51] Wierzbicki, Andrzej. 1984. Models and Sensitivity of Control Systems. Amsterdam: Elsevier Science.

[52] Ronge, Peter. 1985. "Performance Index Sensitivity of Optimal Control Systems with Uncertain Parameters." Optimal Control Applications \& Methods 6: 359-384.

[53] Meinshausen, M., S.C.B. Raper and T.M.L. Wigley. 2011a. Emulating coupled atmosphereocean and carbon cycle models with a simpler model, MAGICC6: Part I - Model Description and Calibration. Atmospheric Chemistry and Physics, 11, 1417--1456. 
[54] Meinshausen, M., S. Smith, K. Calvin, J. Daniel, M. Kainuma, J. F. Lamarque, K. Matsumoto, S. Montzka, S. Raper, K. Riahi, A. Thomson, G. Velders and D. P. van Vuuren. 2011b. The RCP greenhouse gas concentrations and their extensions from 1765 to 2300. Climatic Change, 109(1), 213--241.

[55] Judd, K.L. (1998). Numerical Methods in Economics. The MIT Press.

[56] Cai, Y. 2010. Dynamic Programming and Its Application in Economics and Finance. PhD thesis, Stanford University.

[57] Drud, A.S. 1996. CONOPT: A System for Large Scale Nonlinear Optimization. ARKI Consulting and Development A/S, Bagsvaerd, Denmark.

[58] McCarl, B., et al. 2011. McCarl expanded GAMS user guide, version 23.6. http://www.gams.com/mccarl/mccarlhtml/. Accessed 06 September 2012. 